Transclinic with the services of the PHS, we are able to provide multidisciplinary services, including social support, patient-empowerment, HRT, STI/HIV testing and provision of pre-exposure prophylaxis.

Outcomes/Impact By reaching this previously unsuccessfully engaged population, several public health concerns can be addressed, including prevention and early detection of HIV, improving linkage to care for those living with HIV, prevention of STI transmission and of harm cause by informal HRT. Strong community-led social support encourages patient-empowerment and trust in available medical health services.

Innovation and significance Fusing peer-to-peer organisations with professional healthcare organisations may result in increased acces to key communities and improved health outcomes. Combining services from TUE and the PHS of Amsterdam resulted in the first alliance in the Netherlands between a public health service and a transgender-led organisation.

\subsection{PATHWAYS BETWEEN INTERSECTIONAL STIGMA, GENDER EQUITABLE NORMS, AND CONDOM OUTCOMES AMONG URBAN REFUGEE AND DISPLACED YOUTH IN KAMPALA, UGANDA}

${ }^{1} \mathrm{C}$ Logie*, ${ }^{2} \mathrm{M}$ Okumu, ${ }^{3} \mathrm{~S}$ Mwima, ${ }^{4} \mathrm{P}$ Kyambadde, ${ }^{5} \mathrm{R}$ Hakiza. ${ }^{1}$ University of Toronto, Toronto, Canada; ${ }^{2}$ University of North Carolina Chapel Hill, Chapel Hill, USA; ${ }^{3}$ Uganda Ministry of Health, Kampala, Uganda; ${ }^{4}$ Mulago Hospital, Kampala, Uganda; ${ }^{5}$ Young African Refugees for Integral Development, Kampala, Uganda

10.1136/sextrans-2021-sti.163

Background Social inequities elevate HIV vulnerabilities among youth in humanitarian contexts. Condom efficacy-knowledge, intention, and relationship dynamics that facilitate condom negotiation-is understudied among refugee youth. We examined social-ecological factors (stigma, gender equitable norms [GEN], depression) associated with condom efficacy and use among urban refugee youth in Kampala, Uganda.

Methods We conducted a cross-sectional survey with refugee youth aged 16-24 in Kampala's informal settlements. In multivariable regression analyses we examined associations between adolescent sexual and reproductive health (SRH)-related stigma, HIV-related stigma, and GEN with condom efficacy and recent (past 3-month) consistent condom use among sexually active participants. In path analyses we tested: direct effects of stigma (adolescent SRH-related, HIV-related) and GEN on condom efficacy, and indirect effects via depression; and direct effects of stigma (adolescent SRH-related, HIVrelated) and GEN on recent consistent condom use, and indirect effects via condom efficacy.

Results Among participants (mean age: 19.59, SD: 2.59; women: $n=333$, men: $n=112$ ), $62.5 \%$ were sexually active. Of these, only $53.3 \%$ reported consistent condom use. In multivariable analyses, lower adolescent SRH-related $(\beta=$ $0.18, \mathrm{p}<0.001)$ and HIV-related $(\beta=-0.18, \mathrm{p}<0.001)$ stigma and higher GEN $(\beta=0.15, p<0.001)$ were associated with condom efficacy. Among sexually active participants, GEN was associated with increased (AOR: 1.07, 95\%CI: 1.01-1.13), and adolescent SRH-related stigma with reduced (AOR: 0.92, 95\%CI: 0.84-0.99), odds of recent consistent condom use.
There were direct pathways from lower stigma (adolescent SRH-related, HIV-related) and higher GEN to condom efficacy. Depression partially mediated the pathway from HIVrelated stigma to condom efficacy. Condom efficacy mediated pathways from stigma (HIV-related, adolescent SRH-related) and GEN to consistent condom use.

Conclusion Consistent condom use was low and associated with community (lower stigma, gender equity), interpersonal (condom efficacy), and intrapersonal (reduced depression) factors. Gender transformative and intersectional stigma reduction interventions are needed to advance HIV/STI prevention among urban refugee youth in Kampala.

\section{Late breakers}

\subsection{A CLINICAL ENDOCERVICAL NEISSERIA GONORRHOEAE ISOLATE TRANSIENTLY COLONIZES THE NONHUMAN PRIMATE LOWER GENITAL TRACT}

O Soge ${ }^{*}$, Y Cosgrove-sweeney, R Pascual, D Patton. University of Washington, Seattle, USA

\subsection{6/sextrans-2021-sti.164}

Background The development of a nonhuman primate (NHP) model for gonococcal infection will provide a clinically immunologically relevant model to test future vaccine candidates and novel antimicrobials for multidrug-resistant Neisseria gonorrhoeae (GC). We adapted principles of the established murine model to a NHP model for GC in the pigtailed macaque (Macaca nemestrina).

Methods Twelve Macaca nemestrina underwent nine separate experiments, each designed to assess one or more aspects of NHP model refinement. NHP were exposed to two standard GC strains (FA1090 and MS11) and seven recent clinical GC isolates. Experimental exposure consisted of cervical inoculation(s), administered to Chlamydia trachomatis (CT)-infected and CT-naïve NHP, during different phases of the menstrual cycle, and in the presence or absence of streptomycin treatment. GC detection was performed by culture and nucleic acid amplification testing of cervical secretions during the first three days after experimental challenge, and weekly thereafter.

Results A single clinical isolate (UW-Cx1) transiently colonized the pigtail macaque's lower reproductive tract (GC culture positive to day 3 ; NAAT positive to week 1 ). UW-Cx1, isolated from a clinical endocervical specimen in 2017, is a P1B strain with piliation confirmed at the time of GC challenge. The in vitro growth of UW-Cx1 is not inhibited by macaque cervicovaginal fluid (agar diffusion assay), is highly susceptible to macaque serum complement (bactericidal50 titer: 1.17), and susceptible to cefixime and ceftriaxone. Whole genome sequencing determined that UW-Cx1 and eight sequenced macaque-recovered GC isolates are phylogenetically related. Variables that need to be controlled in the mouse model (estrous cycle stage, suppression of commensal flora) did not affect GC colonization in NHP.

Conclusion We have identified a single clinical GC isolate that has an affinity for the NHP cervicovaginal environment. This strain, UW-Cx1, has repeatedly colonized the macaque cervix, as have the macaque-recovered progeny, when delivered by endocervical challenge. 